\title{
Organic Solvent-Free and Simple Method for Determining Cyromazine and Its Metabolite, Melamine, in Cow’s Milk
}

\author{
Naoto Furusawa \\ Graduate School of Human Life Science, Osaka City University, Osaka, Japan. \\ Email: furusawa@life.osaka-cu.ac.jp
}

Received April 12 $2^{\text {th }}, 2012$; revised May $3^{\text {rd }}, 2012$; accepted May $20^{\text {th }}, 2012$

\begin{abstract}
This paper described an organic solvent-free, rapid, simple, and space-saving method of sample preparation followed by HPLC coupled photo-diode array (PDA) detector for simultaneous quantification of cyromazine (CYR) and its decycropropylated metabolite, melamine (MEL), in milk. The HPLC-PDA was performed on an Inertsil ${ }^{\circledR}$ HILIC column with an isocratic aqueous mobile phase. Analytes were extracted from the sample using water, and purified by Mono$\mathrm{Spin}^{\circledR}-\mathrm{C} 18$, a centrifugal monolithic silica spin mini-columns, and quantified within $20 \mathrm{~min}$. The method, performed under 100\% aqueous conditions, obtained average recoveries for CYR and MEL in the range of $93.2 \%-99.1 \%$ with relative standard deviations $\leq 2.8 \%$. The quantitation limits were $8.5 \mathrm{ng} / \mathrm{mL}$ for CYR and $10 \mathrm{ng} / \mathrm{mL}$ for MEL, respectively. No organic solvents were used at any stage of the analysis.
\end{abstract}

Keywords: Cyromazine; Melamine; HPLC-PDA; Milk: Organic Solvent-Free

\section{Introduction}

Cyromazine (CYR) is a triazine insect growth regulator used as an insecticide. In veterinary medicine, CYR is used as an ectoparasiticide and is added to animal feed to prevent fly from the manure, so as to improve the hygiene control of animal housing environments. CYR can be decycropropylated to melamine (MEL) (Figure 1) in plants and food-producing animals. The biotransformed MEL has been isolated from laying hen, goat, sheep, cow, and their products [1].

The 2008 Chinese milk scandal broke as infants consumed formula containing MEL were falling sick with urinary calculus and kidney damage [2] and the melamine adulteration of a variety of food products has instantly become a global problem. MEL, a nitrogen-rich organic chemical, is added to milk to boost the protein levels, making them appear higher than they actually are. Chronic exposure may lead to reproductive damage, or bladder or kidney stones, which can lead to bladder cancer [3-7].

On October, 2008, FDA issued its Interim Safety and Risk Assessment of MEL in Food for Humans in consideration of the potential public health concerns from foods.

In applying an additional 10 -fold safety factor and using the TDI (Tolerable Daily Intake) of $0.63 \mathrm{mg} / \mathrm{kg}$ b.w./day, the safety/risk assessment concluded that a maximum tolerance levels of MEL below $2.5 \mathrm{ppm}$ in foods other than infant formula do not raise public health concerns [8].

In response the global MEL problem, the United Nations food standards body, the Codex Alimentarius Commission, announced formal international limits for melamine allowed in food and animal feed during its meeting on July, 2010. The maximum amount of MEL allowed in foods and animal feed is $2.5 \mathrm{mg} / \mathrm{kg}$, equal to the FDA limit. The international maximum levels help governments differentiate between low levels of unavoidable melamine occurrence that do not cause health problems, and deliberate adulteration-thereby protecting public health without unnecessary impediments to international trades [9].

Milk contains a good balance of protein, fat and carbohydrate, is an indispensable food because it is inexpensive and readily available. It becomes a raw material of every processed food.
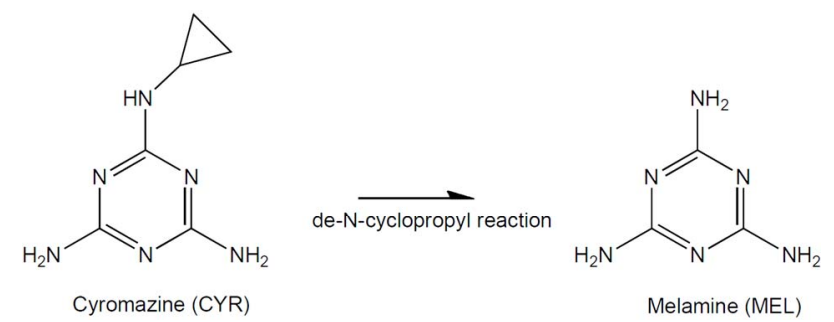

Figure 1. Molecular structures of cyromazine and its decycropropylated metabolite (melamine). 
To ensure that milk for human consumption is residue-free of CYR, the Codex has set a maximum residue limit (MRL) for CYR in milk at $10 \mathrm{ng} / \mathrm{mL}$ [10]. Strict and rapid monitoring the presences of CYR and MEL in milk is, therefore, an important means of guaranteeing the international food safety.

In response to the recent expansion in the internal food trade, the development of international harmonized methods to determine chemical residues in foods is essential to guarantee equitable international trade in these foods and protect health for consumers. Whether in industrial nations or developing countries, an internal harmonized method for residue monitoring in foods is urgently-needed. The optimal harmonized method for the routine monitoring chemicals such as melamine in foods must be simple, small scale, economical in time and cost, and must cause no harm to the environment and humans.

The Food Safety and Inspection Service (FSIS) of the United States Department of Agriculture (USDA) have been provided a test method based on high-performance liquid chromatography (HPLC) for analyzing CYR and MEL in animal tissues in its Chemistry Laboratory Guidebook which contains test methods used by FSIS Laboratories to support the Agency's inspection program, ensuring that meat, poultry, and egg products are safe, wholesome and accurately labeled [11]. Previous techniques based on high-performance liquid chromatography (HPLC) for simultaneous determination of CYR and MEL in animal-derived foods [12-16] has crucial drawbacks: 1) all of methods consume organic solvents in the instrumental analyses as well as for extraction and de-proteinization in sample preparation. The risk associated with these solvents extends beyond direct implications to human health by affecting the ecosystem in which we all reside. Furthermore, the disposal of waste organic solvents through incineration has steadily increasing over the past decade and costs large amounts of money. Thus, eliminating the use of organic solvents and reagents is an important goal in terms of protecting the environment, human health, and the economy $[17,18] .2$ ) most of the recent methods are based on LC-MS/MS. The facility is available are limited to part of industrial nations because these are hugely expensive, and the methodologies use complex and specific. These are unavailable in a lot of laboratories for routine analysis, particularly in developing countries. No optimal method that satisfies the aforementioned requirements has yet been identified.

This article describes an ultrasafe, idiotproof, and inexpensive method to strictly monitor CYR and MEL residues in milk using a $100 \%$ aqueous solution in sample preparation and HPLC separation without organic solvent consumption.

\section{Experimental}

\subsection{Reagents}

Standards of cyromazine (CYR) and melamine (MEL) and other chemicals were purchased from Wako Pure Chem. Ltd. (Osaka, Japan). Distilled water was of HPLC grade. $0.5 \mathrm{moL} / \mathrm{L}$ 1-octanesulfonic acid sodium salt solution (low UV type) was of ion-pair chromatography grade. These standards and chemicals were greater than $99 \%$ purity.

\subsection{Apparatus}

The following apparatuses were used in the sample preparation: handheld ultrasonic-homogenizer (model HOM-100, 2 mm ID probe, Iwaki Glass Co., Ltd., Funabashi, Japan); micro-centrifuge (Biofuge ${ }^{\circledR}$ fresco, Kendo Lab. Products, Hanau, Germany); two types of Mono$\operatorname{Spin}^{\circledR}$ as centrifugal monolithic silica spin mini-columns (sample throughput volume $\leq 300 \mu \mathrm{L}$ ), MonoSpin-C18 (octadecyl and non-polar functional group) and MonoSpin-SCX (bonded propyl benzene sulfone acid combining both strong cation and non-polarity) (GL Sciences, Inc., Tokyo, Japan). The following types of non-polar sorbent columns $\left(5 \mu \mathrm{m} d_{p}, 150 \times 4.6 \mathrm{~mm}\right)$ for HPLC were used: Wakosil 5TMS (C1) (Wako); Mightysil ${ }^{\circledR}$ RP-4GP (C4) (Kanto Chemical Co., Inc., Tokyo, Japan); Mightysil $^{\circledR}$ RP-18 GP Aqua (C18) (Kanto); Inertsil ${ }^{\circledR}$ ODS-4 (C18) (GL Science); Inertsil ${ }^{\circledR}$ HILIC (alkyl diol) (GL Science) (Table 1).

The HPLC system, used for method development, included a model PU-980 pump and DG-980-50-degasser (Jasco Corp., Tokyo, Japan) equipped with a model CO810 column oven (Thosoh Corp., Tokyo, Japan), as well as a model SPD-M10A VP photodiode-array (PDA) detector (Shimadzu Scientific Instruments, Kyoto, Japan). Table 1 lists the particle physical specifications.

\subsection{HPLC Operating Conditions}

The analytical column was an Inertsi ${ }^{\circledR}$ HILIC $(150 \times 4.6$ $\mathrm{mm}, 5 \mu \mathrm{m} d_{p}$ ) column using a $0.05 \mathrm{moL} / \mathrm{L} 1$-octanesulfonic acid mobile phase at a flow rate of $1.0 \mathrm{~mL} / \mathrm{min}$ at $40^{\circ} \mathrm{C}$. PDA detector was operated at $190-300 \mathrm{~nm}$ : the monitoring wavelength was adjusted to $206 \mathrm{~nm}$ which represents an average maximum for the analytes. The injection volume was $20 \mu \mathrm{L}$.

\subsection{Preparation of Stock Standards and Working Mixed Solutions}

Stock standard solutions of CYR and MEL were prepared by dissolving each of CYR and MEL in water to a concentration of $10,000 \mathrm{ng} / \mathrm{mL}$. Working mixed standard solutions of these two compounds were prepared by 
suitably diluting the stock solutions with water. These solutions were kept in a refrigerator $\left(5^{\circ} \mathrm{C}\right)$.

\subsection{Preparation of Calibration Standards and Quality Control Samples}

For method validation studies, calibration standards and quality control samples (QCs), terms defined in the FDA guideline [22], were prepared by spiking appropriate aliquots of the mixed standard solution in blank milk samples. Calibration standards were used to construct calibration curves from which the concentrations of analytes in unknown monitoring samples are determined practically. QCs used to evaluate the performance of the proposed method. In this study, the standards were prepared in the range of $20-1000 \mathrm{ng} / \mathrm{mL}$ for CYR and $20-$ $3000 \mathrm{ng} / \mathrm{mL}$, respectively. Three $\mathrm{QC}$ levels $(\mathrm{QC} 1=30$ $\mathrm{ng} / \mathrm{mL}$ for CYR and $50 \mathrm{ng}$ for MEL; QC2 $=100 \mathrm{ng} / \mathrm{mL}$ for both analytes; QC3 $=500 \mathrm{ng} / \mathrm{mL}$ for CYR and 250 $\mathrm{ng} / \mathrm{mL}$ for MEL) were prepared.

\subsection{Sample Preparation}

An accurate $0.1 \mathrm{~mL}$ sample was taken into a micro-centrifuge tube and homogenized with $0.6 \mathrm{~mL}$ of water with a handheld ultrasonic-homogenizer for $30 \mathrm{~s}$. After being homogenized, the capped tube was centrifuged at 10,000 $\mathrm{g}$ for $5 \mathrm{~min}$. A $0.1 \mathrm{~mL}$ of supernatant liquid was poured to a MonoSpin-C18 and centrifuged at $3500 \mathrm{~g}$ for $1 \mathrm{~min}$. The eluate was injected into the HPLC system.

\subsection{Method Validation}

The performance of the developed method was validated in terms of some parameters from the international guidelines for bio-analytical procedure [19-23]. The quality parameters established were linearity, accuracy, precision, sensitivity, specificity, selectivity, robustness, system suitability.

\section{Results and Discussion}

\subsection{Sample Preparation}

The ultrasonic-homogenization enabled the satisfactory extraction of CYR and MEL from a milk sample with a $100 \%$ water. The author used two types of centrifugal monolithic silica spin mini-columns, MonoSpins (MonoSpin-C18 and-SCX), for the further cleanup technique. The spin mini-column is a monolithic SPE column excellent for the small volume sample with easy and quick operation by centrifuge [24]. A $100 \%$ water was used as the eluent and the recoveries of CYR and MEL from these mini-columns were compared. Since the recoveries of the target compounds from the column vary with centrifugal acceleration (rotary speed), this study was also investigated an optimal acceleration $(\geq 1000 \mathrm{~g})$ to recovery CYR and MEL from the spin mini-columns. A $100 \mu \mathrm{L}$ portion of a mixed standard solution containing $0.5 \mu \mathrm{g}$ of each compound was applied to the spin mini-column. The centrifugal time was standardized at 1 min. MonoSpin-C18 gave satisfactory recoveries ( $\geq 96 \%$ ) and repeatabilities (RSD $\leq 2 \%, n=3$ ) for CYR and MEL when the centrifugal acceleration was $3500 \mathrm{~g}$ for $1 \mathrm{~min}$. The MonoSpin-C18 was therefore used a centrifugal monolithic silica spin mini-column. The present procedure can realize small-scale extractions and easy purifications of CYR and MEL in a short time while completely eliminating the consumption of organic solvents. The procedure resulted in high recoveries and repeatabilities.

\subsection{Optimum HPLC Conditions}

In order to achieve the separation with a $100 \%$ aqueous mobile phase, this study tested five types of non-polar sorbent columns. Table 1 lists the physical and chemical specifications. The author used $0.05 \mathrm{moL} / \mathrm{L} 1$-octanesulfonic acid as the isocratic aqueous mobile phase and examined column temperatures $\geq 25^{\circ} \mathrm{C}$, flow rates $\geq 0.5$

Table 1. Physical/chemical specifications of the reversed-phase columns ${ }^{\text {a }}$ used and chromatographic CYR and MEL separations obtained under the HPLC conditions examined ${ }^{\mathrm{b}}$.

\begin{tabular}{cccccccc}
\hline & \multicolumn{2}{c}{ Column } & $\begin{array}{c}\text { Pore diameter } \\
(\mathrm{nm})\end{array}$ & $\begin{array}{c}\text { Pore volume } \\
(\mathrm{mL} / \mathrm{g})\end{array}$ & $\begin{array}{c}\text { Surface area } \\
\left(\mathrm{m}^{2} / \mathrm{g}\right)\end{array}$ & $\begin{array}{c}\text { Carbon load } \\
(\%)\end{array}$ & $\begin{array}{c}\text { HPLC } \\
\text { separation }\end{array}$ \\
\hline Symbol & Silica (type) & \multicolumn{1}{c}{ Trade name } & 12 & 1.0 & 300 & 4 & Not separated (NS) \\
A & $\mathrm{C} 1$ & Wakosil 5TMS & 12.5 & 1.1 & 350 & 4 & NS \\
B & C4 & Mightysil RP-4 GP & 13.5 & 0.9 & 270 & 15 & NS \\
C & C18 & Mightysil RP-18 GP Aqua & 10 & 1.05 & 450 & 11 & NS \\
D & C18 & Inertsil ODS-4 & 10 & 1.05 & 450 & 20 & Completely separated \\
E & Alkyl diol & Inertsil HILIC &
\end{tabular}

${ }^{\mathrm{a}} 150 \times 4.6 \mathrm{~mm} ; d_{\mathrm{p}}=5 \mu \mathrm{m}$ : data supplied from manufactures; ${ }^{\mathrm{b}}$ Mobile phase of water; column temperatures $\geq 25^{\circ} \mathrm{C}$; flow-rates $\geq 0.5 \mathrm{~mL} / \mathrm{min} ; \mathrm{HPLC}$ retention times $\leq 15 \mathrm{~min} ;{ }^{\mathrm{c}}$ Not separated: between MEL and the interfering milk extract. 
$\mathrm{mL} / \mathrm{min}$, and HPLC retention times $\leq 15 \mathrm{~min}$. Because the HPLC separations were performed serially, the time per run was critical for routine residue monitoring. The short run time not only increased sample throughout for analysis but also affected the method-development time. The seven columns were compared with regard to the separation among CYR, MEL, and their interfering peaks obtained upon injection of equal amounts. The chromatographic separations within the conditions ranges examined are also presented in Table 1.

Columns A-D had difficulty separating MEL and the interfering milk extract throughout the examined condition ranges. An ideal chromatogram with complete separations of CYR, MEL, and interfering peaks, and their short retention times was obtained by a Column $\mathrm{E}$ and a $0.05 \mathrm{~mol} / \mathrm{L} 1$-octanesulfonic acid mobile phase with a column temperature of $40^{\circ} \mathrm{C}$ and a flow rate of 1.0 $\mathrm{mL} / \mathrm{min}$. The HPLC system achieved optimal separation $<6.5$ min without the need for a gradient system to improve the separation. The ion-pair action of 1-octanesulfonic acid mobile phase used here was necessary to obtain the findings described.

Figure 2 displays that the resulting chromatograms were free of interfering compounds for quantitation and identification of CYR and MEL by HPLC, with PDA detector set at $206 \mathrm{~nm}$ (giving an average maximum for CYR and MEL). This figure demonstrates that the present method can provide the quantitation and identification of CYR and MEL.

\subsection{Method Validation}

\subsubsection{Main Quality Parameters}

Table 2 summarizes the values obtained for the main parameters. The accuracy and precision are well within the international method acceptance criteria $[19,21,23]$. The QL for CYR $(9 \mathrm{ng} / \mathrm{mL})$ was lower than the MRL of $10 \mathrm{ng} / \mathrm{mL}$ [10]. The other validation findings are as follows:

\subsubsection{Specificity and Selectivity}

The application of the proposed procedure to 10 blank milk samples from different species (Holstein-Friesian and Jersey) demonstrated that no interference peak was presented around the retention times for CYR and MEL in any of the sample examined.

The present HPLC-PDA system easily confirmed the peak identity of target compound. CYR and MEL were identified in a milk sample by their retention times and absorption spectra. The CYR and MEL spectra obtained from the milk sample were practically identical to those of the standards. Because of the complete separations and the high absorbance of CYR and MEL, PDA detection at trace levels is fully available. It is, therefore, instructive to demonstrate purification effectiveness of the sample preparation. The system did not require the use of MS, which is very expensive and is not available in a lot of laboratories for routine analysis, particularly in developing countries.

\subsubsection{Robustness}

Some HPLC parameters were performed using a spiked (100 ng/mL of each compound) milk sample obtained under the established procedure.

Changes of $\pm 5 \%$ units of the flow rate $(1.0 \mathrm{~mL} / \mathrm{min})$ and the column temperature $\left(40^{\circ} \mathrm{C}\right)$ were determined. The effect on the peak areas and the validations in the retention times were evaluated. Changes of $\pm 5 \%$ of the flow rate and the column temperature had no effect on the peak areas, whereas the variations in the retention

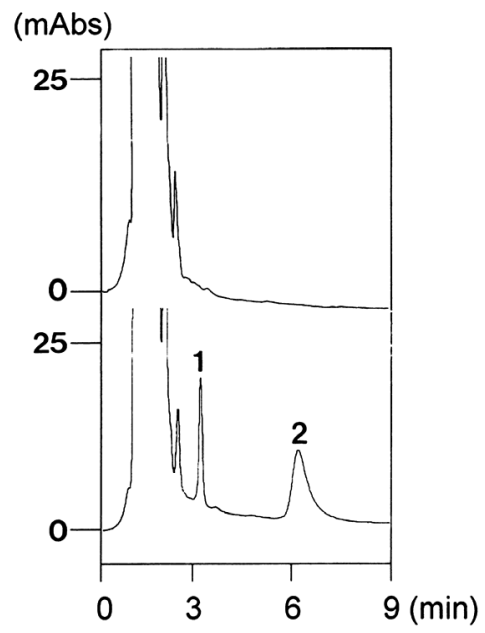

Figure 2. Chromatograms obtained from the HPLC system for a blank milk sample (upper profile) and a milk sample spiked with CYR (500 ng/mL) and MEL (250 ng/mL) (lower profile). The PDA detector was set at $206 \mathrm{~nm}$. Peaks, $1=$ MEL (retention time, $\left.R_{t}=3.08 \mathrm{~min}\right) ; 2=\operatorname{CYR}\left(\mathrm{R}_{\mathrm{t}}=6.23\right.$ min).

Table 2. The main method validation data.

\begin{tabular}{ccc}
\hline Paramer & CYR & MEL \\
\hline${\text { Linearity }\left(\mathrm{r}^{\mathrm{a}}\right)}^{\mathrm{a}}$ & 0.991 & 0.994 \\
Accuracy $^{\mathrm{b}}$ & $93.2-97.8$ & $94.5-99.1$ \\
Precision $^{\mathrm{c}}$ & $\leq 2.8$ & $\leq 2.4$ \\
Sensitivity $^{\mathrm{d}}$ & 8.5 & 10 \\
\hline
\end{tabular}

${ }^{{ }^{a}} \mathrm{r}$ is the correlation coefficient $(\mathrm{P}<0.01)$ : mean of three determinations using spiked milk samples for calibration curves: ranges of concentrations were $20-1000 \mathrm{ng} / \mathrm{mL}$ for CYR and $20-3000 \mathrm{ng} / \mathrm{mL}$ for MEL, respectively; ${ }^{\mathrm{b}}$ Average recoveries (\%) from six replicates at three $\mathrm{QC}$ levels $(\mathrm{QC} 1=30$ $\mathrm{ng} / \mathrm{mL}$ for CYR and $50 \mathrm{ng}$ for MEL; QC2 $=100 \mathrm{ng} / \mathrm{mL}$ for both analytes; $\mathrm{QC} 3=500 \mathrm{ng} / \mathrm{mL}$ for CYR and $250 \mathrm{ng} / \mathrm{mL}$ for MEL); ${ }^{\mathrm{c}}$ Values are RSDs (\%, $\mathrm{n}=6$ of each level); ${ }^{\mathrm{d}}$ Quantitation limit $(\mathrm{ng} / \mathrm{mL}), \mathrm{QL}$ as the concentration of analyte giving a signal-to-noise ratio $>10$ 
times were obtained with the flow rate and the column temperature. Normal retention times for CYR and MEL were 6.23 and $3.08 \mathrm{~min}$, respectively. At $+5 \%$ the flow rate, the two retention times were decreased, ranging between 1.6 and $5.7 \%$ and at $-5 \%$, the times were increased ranging between 5.5 and $8.0 \%$. By changing the column temperature by $+5 \%$, decreasing retention times obtained were $1.9 \%-7.5 \%$, however, no significant variations were observed with $-5 \%$. During these studies, all the target compounds were separated.

\subsubsection{System Suitability}

The system-suitability evaluation is an essential parameter of HPLC determination, and it ascertains the strictness of the system used. The suitability was evaluated as the relative standard deviations of peak areas and retention times calculated for 20 replicate injections of a spiked milk sample ( $100 \mathrm{ng} / \mathrm{mL}$ of each compound). The values for CYR and MEL were estimated to be $<0.5 \%$ for peak areas and $<0.8 \%$ for retention times, respectively.

\subsection{Cost and Time Performance}

The total time and budget required for the analysis of a single sample was $<20 \mathrm{~min}$ and approximately US $\$ 6.2$ as of April 12, 2012, respectively. For sequential analyses, a batch of 24 samples could be analyzed in $4 \mathrm{~h}$. These findings became term required for an international harmonized residue analysis. The organic solvent-free and short analytical time not only increased the sample throughput for analysis but also positively affected the cost and environmental/human impacts.

\subsection{Application to Residue Monitoring in Commercial Samples}

Milk was purchased from a number of convenience stores in Osaka, Japan, used as real milk samples and analyzed using the proposed method. No samples contained detectable concentrations of CYR and MEL. The resulting chromatograms were free from interference.

\section{Conclusion}

An organic solvent-free method does not use organic solvents for simultaneous determination of CYR and MEL in milk has been successfully developed and validated. The present procedure provided an easy-to-use, rapid, space-saving, and non-use of organic solvents and resulted in high recovery and repeatability with considerable saving of analysis time/cost. The procedure may be proposed as an international harmonized analytical method for the routine monitoring of CYR and MEL in milk.

\section{REFERENCES}

[1] FAO, Pesticide Management, JMPR Evaluation, List of Pesticides Evaluated by JMPR and JMPS, Cyromazine, JMPR No. 169, 2009 (R) Evaluation.

http://www.fao.org/agriculture/crops/core-themes/theme/ pests/pm/lpe/lpe-c/en/

[2] A. K. Hau, T. H. Kwan and P. K. Li, "Melamine Toxicity and the Kidney," Journal of the American Society Nephrology, Vol. 20, 2009, pp. 245-250. doi:10.1681/ASN.2008101065 http://jasn.asnjournals.org/content/20/2/245.full.pdf + html

[3] MSDS. http://msds.chem.ox.ac.uk/index $2 . h t m l$

[4] International Chemical Safety Card. http://www.cdc.gov/niosh/ipcsneng/neng1154.html

[5] OSHA, "Chemical Sampling Information." http://www.osha.gov/dts/chemicalsampling/data/CH_250 440.html

[6] WHO, "Some Chemicals That Care Tumors of the Kidney or Urinary Bladder in Rodents and Some Other Substances."

http://en.wikipedia.org/wiki/Melamine\#cite_note-melami nemsds-17

[7] H. D. Heck and R. W. Tyl, "The Induction of Bladder Stones by Terephthalic Acid, Dimethyl Terephthalate, and Melamine (2,4,6-Triamino-s-triazine) and Its Relevance to Risk Assessment," Regulatory Toxicology and Pharmacology, Vol. 5, No. 3, 1985, pp. 294-313. doi:10.1016/0273-2300(85)90044-3

[8] FDA, "Protecting and Promoting Your Health," http://www.fda.gov/Food/FoodSafety/FoodContaminants Adulteration/ChemicalContaminants/Melamine/ucm 164520.htm

[9] WHO, Media Centre, International Experts Limit Melamine Levels in Food.

http://www.who.int/mediacentre/news/releases/2010/mela mine food 20100706/en/index.html

[10] Codex, Resticide Residues in Food and Feed, Cyromazine, http://www.codexalimentarius.net/pestres/data/pesticides/ details.html?id=169

[11] USDA FSIS, “Cyromazine and Melamine," July 1991, Retrieved 27 April 2007. http://www.fsis.usda.gov/ophs/clg/Cyromazine.pdf

[12] S. Tittlemier, Health Canada, "Background Paper on Methods for the Analysis of Melamine and Related Compounds in Foods and Animal Feeds," Ottawa, 1-4 December 2008

http://www.who.int/foodsafety/fs_management/Melamine _1.pdf

[13] H. A. Yu, Y. F. Tao, D. M. Chen, Y. L. Wang, Z. Y. Liu, Y. H. Pan, L. L. Huang, D. P. Peng, M. H. Dal, Z. L. Liu and Z. H. Yuan, "Development of a High Performance Liquid Chromatography Method and Liquid Chromatography-Tandem Mass Spectrometry Method with Pressurized Liquid Extraction for Simultaneous Quantification and Confirmation of Cyromazine, Melamine and Its Metabolites in Foods of Animal Origin," Analytica Chimica Acta, Vol. 682, No. 1-2, 2010, pp. 48-58. 
doi:10.1016/j.aca.2010.09.032

[14] S. S. Wang, D. M. Li, Z. D. Hua and M. P. Zhao, "Molecularly Imprinted Monolith Coupled On-Line with High Performance Liquid Chromatography for Simultaneous Quantitative Determination of Cyromazine and Melamine," Analyst, Vol. 136, No. 18, 2011, pp. 36723679. doi:10.1039/c1an15086c

[15] H. W. Sun, L. X. Wang, N. Liu, F. X. Qiao and S. X. Liang, "SPE Then RP-LC for Simultaneous Analysis of Cyromazine and Its Metabolite Melamine in Liquid Milk and Egg," Chromatographia, Vol. 70, No. 11, 2009, pp. 1685-1689. doi:10.1365/s10337-009-1355-x

[16] R. C. Wei, R. Wang, Q. F. Zeng, M. Chen and T. Z. Lui, "High-Performance Liquid Chromatographic Method for the Determination of Cyromazine and Melamine Residues in Milk and Pork," Journal of Chromatographic Science, Vol. 47, 2009, pp. 581-584.

[17] P. T. Anastas and J. C. Warner, "Green Chemistry: Theory and Practice," Oxford University Press, Oxford, 1998.

[18] T. Yoshimura, T. Nishinomiya, Y. Honda and M. Murabayashi, "Green Chemistry-Aim for the Zero EmissionChemicals," Sankyo Publishing Co. Ltd. Press, Tokyo, 2001.
[19] L. Huber (Ed.), "Validation and Quantification in Analytical Laboratories," Interpharm Press, East England, 1998.

[20] ICH, "Work Products, ICH Guidelines, Quality Guidelines." http://www.ich.org/products/guidelines.html

[21] AOAC International, "Method Validation, Guidelines \& References.” http://www.aoac.org/vmeth/guidelines.htm

[22] FDA/CDER/CVM, "Guidelines for Industry-Bioanalytical Method Validation," 2001. http:/www.fda.gov/downloads/Drugs/GuidanceComplian ceRegulatoryInformation/Guidances/UCM070107.pdf

[23] Codex Alimentarius Commission, ALINORM 01/23, Codex Committee on Methods of Analysis and Sampling, Joint FAO/WHO Food Standards Programme, the 23rd Section of the Codex Committee on Method of Analysis and Sampling. http://www.codexalimentarius.net/web/archives.jsp?year= 01

[24] GL Sciences, Monolithic SPE Column for the Purification and Enrichment of Small Amount Sample, MonoSpin $^{\circledR}$ Series.

http://www.glsciences.com/products/monolithic products /mono_spin.pdf 